POS PROCEEDINGS

\title{
Measurement of the Higgs boson coupling properties in the diphoton and $Z Z$ decay channels using the ATLAS detector
}

\author{
Ruchi Gupta*† \\ Southern Methodist University (US) \\ E-mail: ruchi.gupta@cern.ch
}

The coupling properties of the Higgs boson are studied in the diphoton and $Z Z$ to four-lepton decay channels using $36.1 \mathrm{fb}^{-1}$ of $p p$ collision data from the LHC at a centre-of-mass energy of $13 \mathrm{TeV}$ collected by the ATLAS detector. Measurements of simplified template cross sections, designed to measure the different Higgs boson production processes in specific regions of phase space, are reported for diphoton and four-leptons decay channels. Cross sections for different Higgs boson production modes are interpreted in terms of coupling modifiers. In the ZZ decay channel, the tensor structure of the Higgs boson couplings is studied using an effective Lagrangian approach for the description of interactions beyond the Standard Model.

The European Physical Society Conference on High Energy Physics

5-12 July

Venice, Italy

${ }^{*}$ Speaker.

${ }^{\dagger}$ On behalf of the ATLAS Collaboration. 


\section{Introduction}

The observation of the Higgs boson by the ATLAS and CMS experiments at centre-of-mass energies of $\sqrt{\mathrm{s}}=7 \mathrm{TeV}$ and $8 \mathrm{TeV}$ has been a major step towards the understanding of the mechanism of electroweak (EW) symmetry breaking. Further measurements of the spin, parity and couplings of the new particle have shown no significant deviation from the predictions for the Standard Model (SM) Higgs boson

The measurement of the Higgs boson coupling properties is performed in diphoton $(H \rightarrow \gamma \gamma)$ and $Z Z\left(H \rightarrow Z Z^{*} \rightarrow 4 \ell\right.$, where $\ell \equiv e$ or $\left.\mu\right)$ decay channels using $36.1 \mathrm{fb}^{-1}$ of Run-2 $p p$ collision data collected by the ATLAS experiment at a centre-of-mass energy of $13 \mathrm{TeV}[1,2]$.

\section{Analysis Strategy}

The ATLAS detector is described in detail in Ref. [3]. Details of the reconstruction and identification of physics objects as well as event selection criteria used are given in Refs. $[1,2]$.

The lepton four-momenta of the leading lepton pair are recomputed by means of a kinematic fit constrained with a $Z$-mass-constraint. The fit uses a Breit-Wigner $Z$ line shape, and a single Gaussian function per lepton to model the momentum resolution function for the expected resolution of each lepton. The expected and observed four-lepton invariant mass distribution of the selected Higgs boson candidates after the event selection with a constrained $Z$ boson mass is shown in Figure 1(a).

The observed invariant mass distribution of the selected diphoton pairs of all categories is shown in Figure 1(b). For illustration purposes, events in each category are weighted according to the expected signal $\left(S_{90}\right)$ to background $\left(B_{90}\right)$ ratio in a $m_{\gamma \gamma}$ region containing $90 \%$ of the expected signal yield, using a weight of the form $\ln \left(1+S_{90} / B_{90}\right)$.

The cross sections for different Higgs boson production processes are measured in several exclusive regions of phase space (Simplified Template Cross Sections [4]). This is done to maximize sensitivity to different production modes for theoretical interpretation, leading to reduced theoretical uncertainties on the results. The cross sections for different production modes are termed as Stage- 0 . The Stage- 0 cross sections are further divided into several phase space bins (called Stage1) for enhanced sensitivity. Due to lack of statistics, some of the Stage-1 categories are merged resulting in reduced Stage-1 cross section measurements [1, 2].

The cross-section results by production mode (Stage-0) are also interpreted in the $\kappa$ framework [5] in which coupling modifiers, $\kappa_{i}$, are introduced to parameterize possible deviations from the SM predictions of the Higgs boson couplings to SM bosons and fermions. One interesting benchmark allows two different Higgs boson coupling strength modifiers, one for fermions and the other for bosons, reflecting the different structure of the interactions of the SM Higgs sector with gauge bosons and fermions. The universal coupling-strength scale factors $\kappa_{F}$ for all fermions and $\kappa_{V}$ for all vector bosons are defined as $\kappa_{V}=\kappa_{W}=\kappa_{Z}$ and $\kappa_{F}=\kappa_{t}=\kappa_{b}=\kappa_{c}=\kappa_{\tau}=\kappa_{\mu}=\kappa_{g}$. It is assumed that there are no undetected or invisible Higgs boson decays. The tensor structure of the Higgs boson couplings is studied in $H \rightarrow Z Z^{*} \rightarrow 4 \ell$ events, probing for admixtures of CP-even and CP-odd interactions in theories beyond the SM (BSM) in addition to the corresponding SM interactions. The analyses are performed assuming that the studied resonance is a single particle 


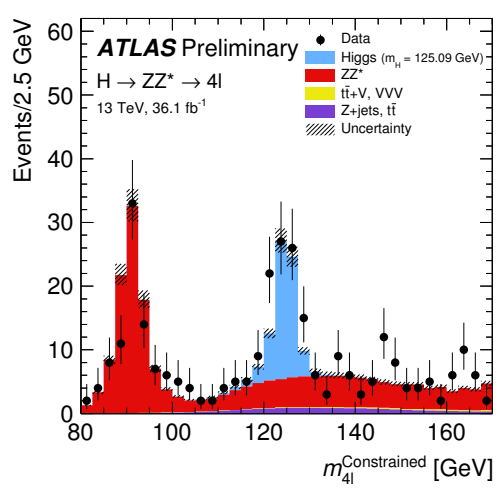

(a)

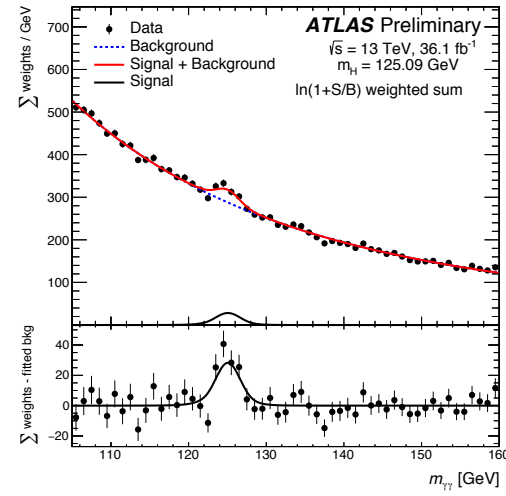

(b)

Figure 1: The observed and expected distribution for (a) four-lepton invariant mass [1] and (b) diphoton invariant mass [2], for the selected Higgs boson candidates, shown for an integrated luminosity of $36.1 \mathrm{fb}^{-1}$ at $\sqrt{\mathrm{s}}=13 \mathrm{TeV}$ assuming the SM Higgs boson signal with a mass $m_{H}=$ $125.09 \mathrm{GeV}$. The error bars represent $68.3 \%$ central confidence interval statistical uncertainty. The dashed band in (a) shows the systematic uncertainty in the prediction. The solid red curve in (b) shows the fitted signal-plus-background model with dotted blue curve showing the background component.

state with spin- 0 and a mass of $125.09 \mathrm{GeV}$ based on experimental results obtained with the LHC Run-1 data $[6,1]$.

\section{Results from $H \rightarrow Z Z^{*} \rightarrow 4 \ell$}

The observed values of $\sigma \cdot B\left(H \rightarrow Z Z^{*}\right)$ and the SM expected cross section in each stage- 0 and reduced Stage-1 production bin are shown in Figure 2.

All Stage- 0 and reduced Stage- 1 ggF measurements agree with the predictions for the SM Higgs boson within $1 \sigma$. Somewhat worse agreement is obtained for the VBF bins due to an observed excess of events in the two VBF-enriched reconstructed event categories. The largest deviation of $2.2 \sigma$ is observed for the Stage- 0 VBF production bin due to an observed excess of events characterized by the presence of at least two jets and a dijet invariant mass above $120 \mathrm{GeV}$. Due to the limited number of events in the $V H$ - and $t t H$-enriched categories, only upper limits are set on the cross sections and signal strengths for these production modes. The $V H$ and $t \bar{t} H$ parameters of interest are constrained to positive values to avoid the fit's prediction of negative total event yields in the $V H$-Lep-enriched and $t t H$-enriched categories and provide a stable fit configuration. It was found that the impact of this constraint on the final fit results is negligible.

Figure 3(a) shows the likelihood contours in the $\left(\sigma_{g g F} \cdot B, \sigma_{V B F} \cdot B\right)$ plane. The compatibility with respect to the Standard Model expectation is at the level of 2.3 $\sigma$. The observed likelihood contours in the $\kappa_{V}-\kappa_{F}$ plane are shown in Figure 3(b) (only the quadrant $\kappa_{F}>0$ and $\kappa_{V}>0$ is shown since this channel is not sensitive to the relative sign of the two coupling modifiers). The compatibility with the Standard Model expectation is at the level of $1.4 \sigma$. Better agreement is observed here compared to the likelihood contours for the cross-section ratios, since the $V H$ production is 


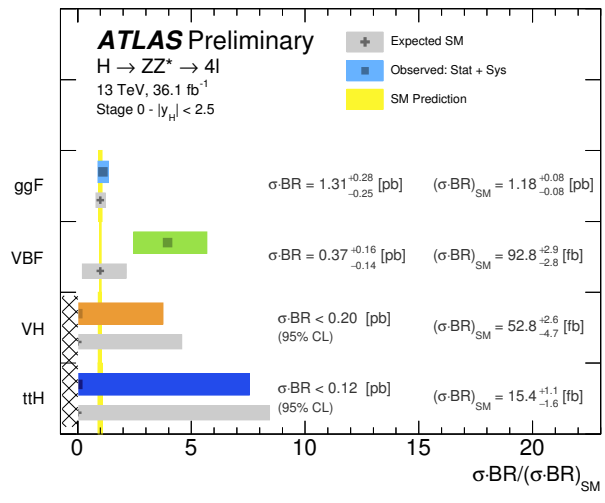

(a)

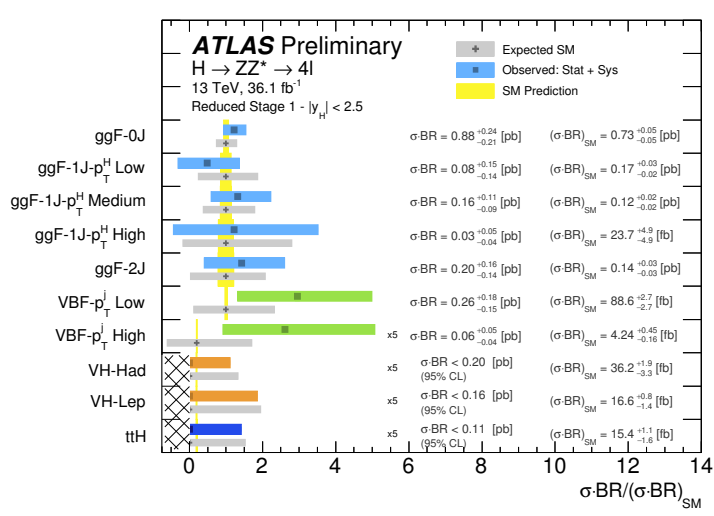

(b)

Figure 2: The observed and expected SM values of the cross-section ratios $\sigma \cdot B$ normalized by the SM expectation $(\sigma \cdot B)_{\text {SM }}$ for the (a) Stage- 0 and (b) reduced Stage-1 production bins for an integrated luminosity of $36.1 \mathrm{fb}^{-1}$ at $\sqrt{\mathrm{s}}=13 \mathrm{TeV}$. Different colours for the observed results indicate different Higgs boson production modes. The hashed area indicates that the $V H$ and $t \bar{t} H$ parameters of interest are constrained to positive values. For visualization purposes, the VBF- $p_{\mathrm{T}}^{j}$-High value and the limits for the three reduced Stage- 1 production bins $V H$-Had, $V H$-Lep and $t t H$ are divided by a factor of five when shown normalized to $(\sigma \cdot B)_{\mathrm{SM}}$. The yellow vertical band represents the theory uncertainty in the signal prediction, while the horizontal grey bands represent the expected measurement uncertainty [1].

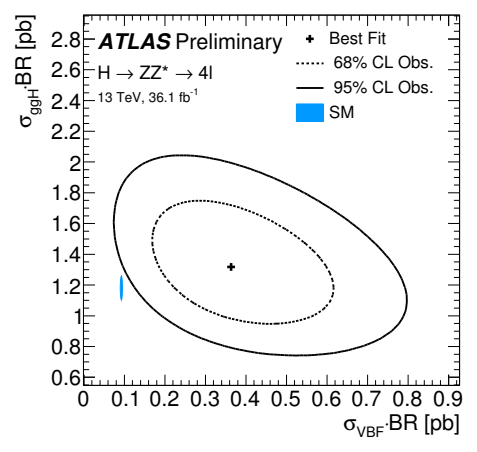

(a)

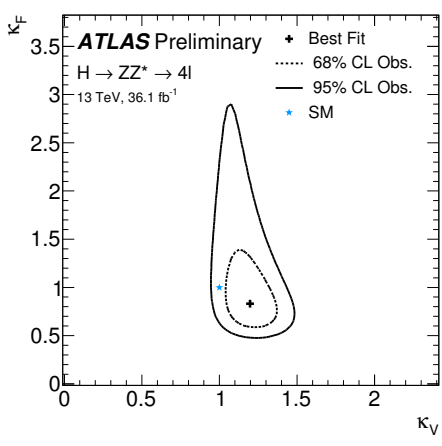

(b)

Figure 3: (a) Likelihood contours at 68\% CL (dashed line) and 95\% CL (solid line) in the $\left(\sigma_{g g F} \cdot B\right.$ , $\left.\sigma_{V B F} \cdot B\right)$ plane and (b) likelihood contours in the $\kappa_{V}-\kappa_{F}$ plane. The best fits to the data (solid cross) and the SM predictions are indicated. In (a), the SM prediction is shown together with its theory uncertainty (filled blue ellipse), while in (b) only the central value of the SM prediction (solid blue star) is shown [1]. 
no longer profiled and the lower observed yield in the $V H$-Lep-enriched categories compared with SM expectations compensates for the observed excess in the VBF categories.

The tensor sturcture of Higgs boson couplings to vector bosons is studied using effective field theory approach as explained in Ref. [1]. In this analysis, the effective Lagrangian terms with coupling parameters $\kappa_{H V V}, \kappa_{A V V}$ and $\kappa_{A g g}$ are considered as possible BSM admixtures to the corresponding SM interactions. These terms describe the CP-even (scalar) and CP-odd (pseudo-scalar) BSM interaction with vector bosons and the CP-odd BSM interaction with gluons, respectively. The BSM couplings are assumed to be the same for $W$ and $Z$ bosons (i.e. $\kappa_{H W W}=\kappa_{H Z Z} \equiv \kappa_{H V V}$ and $\left.\kappa_{A W W}=\kappa_{A Z Z} \equiv \kappa_{A V V}\right)$.

The coupling parameter $\kappa_{A g g}$ is measured assuming that all other BSM couplings are equal to zero. The coupling parameters $\kappa_{H V V}$ and $\kappa_{A V V}$ are probed both simultaneously and $\kappa_{A g g}$ at a time assuming that all other BSM couplings vanish. If not stated otherwise, the SM couplings $\kappa_{\mathrm{SM}}$ and $\kappa_{H g g}$ are fixed to the SM value of one. The BSM changes in the Higgs sector are assumed not to affect the SM background processes. Figure 4(a) shows the observed negative log-likelihood as function of $\kappa_{A g g}$, together with the expectation for the SM Higgs boson. The event yields measured in the introduced reconstructed event categories do not provide any sensitivity to the sign of the $\kappa_{A g g}$ and $\kappa_{A V V}$ coupling parameters.

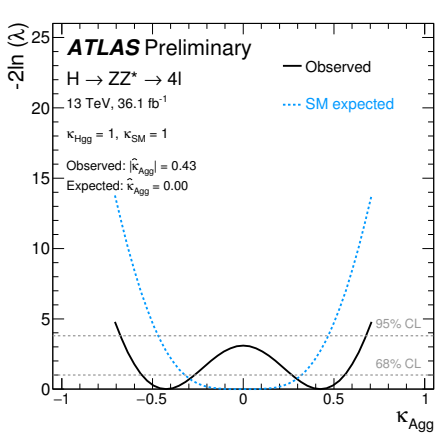

(a)

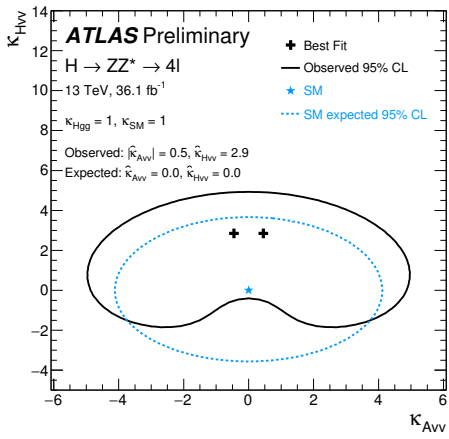

(b)

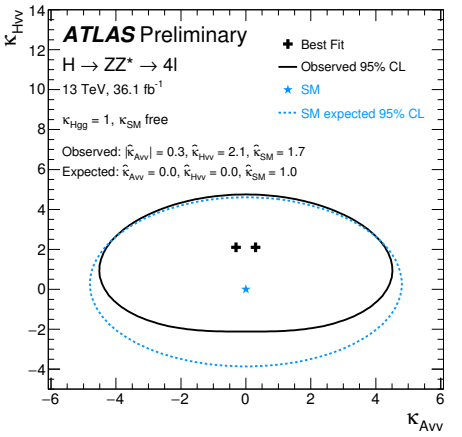

(c)

Figure 4: (a) Observed (black) and SM expected (blue) negative log-likelihood scans for $\kappa_{A g g}$. The horizontal lines indicate the value of the profile likelihood ratio corresponding to the $68 \%$ and 95\% CL intervals for the parameter of interest, assuming the asymptotic $\chi^{2}$ distribution of the test statistic. (b) and (c) : Observed (black) and SM expected (blue) contours of the two-dimensional negative log-likelihood at $95 \%$ CL for the $\kappa_{H V V}$ and $\kappa_{A V V}$ coupling parameters where, $\kappa_{\mathrm{SM}}$ is fixed to the SM value of one in (b) and left as a free parameter of the fit in (c). $36.1 \mathrm{fb}^{-1}$ of data at $\sqrt{\mathrm{s}}=13 \mathrm{TeV}$ is used [1].

The CP-even and CP-odd BSM couplings to heavy vector bosons are also probed simultaneously in a two-dimensional contour analysis of the negative log-likelihood. The results are shown in Figure 4(b) and 4(c). The obtained result is compatible with the SM prediction within $2 \sigma$.

\section{Results from $H \rightarrow \gamma \gamma$}

The signal strengths, calculated as the ratios of the measured Higgs boson production cross sections times diphoton branching ratio to the SM predictions for each production mode, are evaluated by exploiting the sensitivities of the analysis categories to specific production processes, and 
results are shown in Figure 5. The ggH signal strength is $1 \sigma$ below the expectation from the Standard Model, while the VBF signal strength is $2.2 \sigma$ above the prediction. Since no significant evidence is observed for $V H$ and top-quark associated production, the associated production with $Z$ or $W$ bosons is assumed to be affected by a single signal strength parameter (i.e. $\mu_{\mathrm{ZH}}=\mu_{\mathrm{WH}}=\mu_{\mathrm{VH}}$ ). Additionally, the $b \bar{b} H$ contributions are scaled with ggH (i.e. $\left.\mu_{\mathrm{bbH}}=\mu_{\mathrm{ggH}}\right)$, and the sum of $t H$ and $t \bar{t} H$ productions are measured together rather than separately $\left(i . e . \mu_{\mathrm{top}}=\mu_{\mathrm{ttH}+\mathrm{tH}}\right)$.

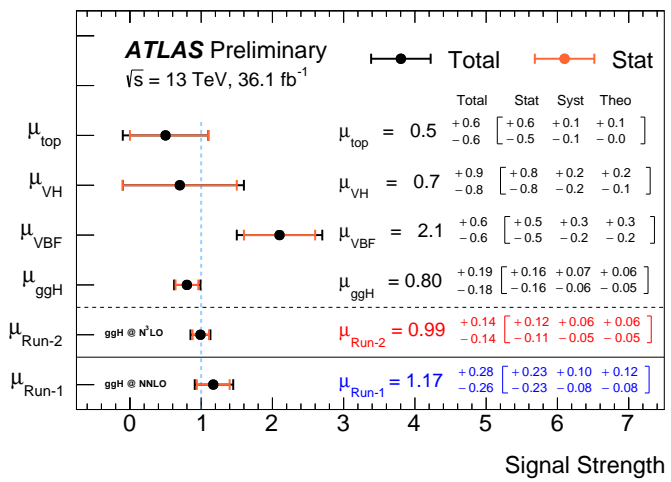

Figure 5: Summary of the signal strengths measured for the different production processes ( $\mathrm{ggH}$, VBF, $V H$ and $t \bar{t} H+t H)$ and globally ( $\mu_{\text {Run-2 }}$ ), compared to the global signal strength measured at 7 and $8 \mathrm{TeV}\left(\mu_{\mathrm{Run}-1}\right)$. The black (red) error bar shows the total (statistical) uncertainty [2].

The $68 \%$ and $95 \% \mathrm{CL}$ two-dimensional contours of $\sigma_{\mathrm{ggH}} \times B(H \rightarrow \gamma \gamma)$ and $\sigma_{\mathrm{VBF}} \times B(H \rightarrow \gamma \gamma)$ are shown in Figure 6(a). The SM expectation of $\sigma_{\mathrm{ggH}} \times B(H \rightarrow \gamma \gamma)$ vs $\sigma_{\mathrm{VBF}} \times B(H \rightarrow \gamma \gamma)$ is within the $95 \%$ CL contour of this measurement.

The effective coupling-strength modifiers, $\kappa_{g}$ and $\kappa_{\gamma}$ describe the loop processes for ggH production and $H \rightarrow \gamma \gamma$ decay. This is possible because BSM particles that might be present in these loops are not expected to appreciably change the kinematics of the corresponding process. The $g g \rightarrow H$ and $H \rightarrow \gamma \gamma$ loop processes can thus be studied through these effective coupling-strength modifiers, providing sensitivity to potential BSM particles in the loops. The 68\% and 95\% CL two-dimensional contours of the effective couplings $\kappa_{g}$ and $\kappa_{\gamma}$ are shown in Figure 6(b), where the $\kappa_{V}$ and $\kappa_{F}$ parameters affecting other production modes are fixed to the SM assumption. In a second model, the ggH and $H \rightarrow \gamma \gamma$ loops are resolved in terms of $\kappa_{V}$ and $\kappa_{F}$. The $68 \%$ and $95 \%$ CL two-dimensional contours of $\kappa_{F}$ and $\kappa_{V}$ are shown in Figure 6(c). The SM prediction is found within the $68 \% \mathrm{CL}$ contour for the former scenario and within the $95 \% \mathrm{CL}$ contour for the latter one.

\section{Conclusions}

The coupling properties of the Higgs boson are studied in the diphoton and four-lepton decay channel using $36.1 \mathrm{fb}^{-1}$ of LHC $p p$ collision data at $\sqrt{\mathrm{s}}=13 \mathrm{TeV}$ collected by the ATLAS experiment. Measurements of simplified template cross sections, designed to measure the different Higgs boson production processes in specific regions of phase space, are reported. The cross sections measured in dedicated production bins are in agreement with the SM predictions.

A deviation of $2.2 \sigma$ is observed for the VBF production in the four-lepton channel due to an observed excess of events characterized by the presence of at least two jets and a dijet invariant 


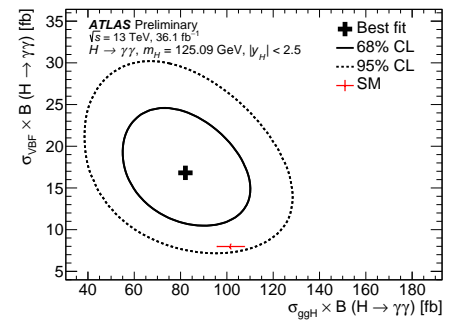

(a)

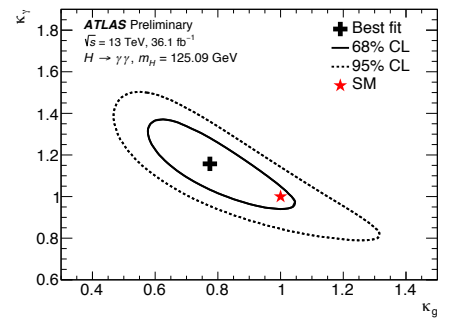

(b)

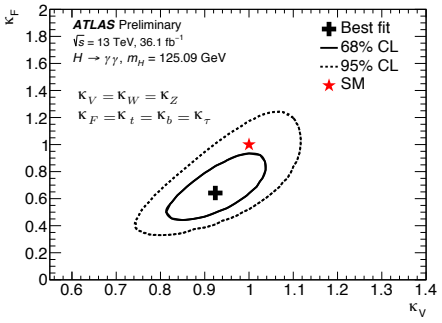

(c)

Figure 6: Likelihood contours in (a) the $\left(\sigma_{\mathrm{ggH}} \times B(H \rightarrow \gamma \gamma), \sigma_{\mathrm{VBF}} \times B(H \rightarrow \gamma \gamma)\right)$ plane (b) the $\left(\kappa_{g}, \kappa_{\gamma}\right)$ plane, and (c) the $\left(\kappa_{V}, \kappa_{F}\right)$ plane, compared to the Standard Model prediction (red star) for a Higgs boson mass $m_{H}=125.09 \mathrm{GeV}$. In (b), the coupling strength parameters $\kappa_{F}$ and $\kappa_{V}$ are assumed to be equal to one. In (c), the coupling strength parameters $\kappa_{g}$ and $\kappa_{\gamma}$ are treated as a function of the more fundamental coupling-strength parameters $\kappa_{F}$ and $\kappa_{V}$ for the particles that contribute to the SM loop processes inducing the Higgs boson couplings to gluons and photons [2].

mass above $120 \mathrm{GeV}$.

The signal strength measurements for various production modes are carried out for the diphoton channel. The global signal strength measurement of $0.99 \pm 0.14$ in diphoton channel improves on the precision of the previous ATLAS measurement by a factor of 2, and $\mathrm{ggH}$ (VBF) production is measured to be $1 \sigma$ below ( $2 \sigma$ above) the Standard Model expectation.

Results are also interpreted in terms of coupling modifiers $\kappa_{V}$ and $\kappa_{F}$, showing compatibility with the SM. Based on event yields observed in each reconstructed event category, constraints are placed on possible BSM interactions of the Higgs boson within the framework of an effective Lagrangian extension of the SM. The data are shown to be consistent with the SM hypothesis, with the largest deviations of about $2 \sigma$ due to the mentioned excess of observed events. Exclusion limits are set on the CP-even and CP-odd BSM couplings of the Higgs boson to vector bosons and on the CP-odd BSM Higgs boson coupling to gluons.

\section{References}

[1] ATLAS Collaboration, Measurement of the Higgs boson coupling properties in the $H \rightarrow$ $Z Z^{*} \rightarrow 4 \ell$ decay channel at $\sqrt{s}=13 \mathrm{TeV}$ with the ATLAS detector, ATLAS-CONF-2017043.

[2] ATLAS Collaboration, Measurements of Higgs boson properties in the diphoton decay channel with $36.1 \mathrm{fb}^{-1} \mathrm{pp}$ collision data at the center-of-mass energy of $13 \mathrm{TeV}$ with the ATLAS detector, ATLAS-CONF-2017-045.

[3] ATLAS Collaboration, The ATLAS Experiment at the CERN Large Hadron Collider, JINST 3 (2008) S08003.

[4] LHC Higgs Cross Section Working Group (D. de Florian et al.), Handbook of LHC Higgs Cross Sections: 4. Deciphering the Nature of the Higgs Sector, (2016), arXiv: 1610.07922 [hep-ph]. 
[5] LHC Higgs Cross Section Working Group, https : / twiki.cern.ch/twiki/bin/ view/LHCPhysics/LHCHXSWG2KAPPA.

[6] ATLAS and CMS Collaborations, Combined Measurement of the Higgs Boson Mass in pp Collisions at $\sqrt{s}=7$ and 8 TeV with the ATLAS and CMS Experiments, Phys. Rev. Lett. 114 (2015) 191803, arXiv: 1503.07589 [hep-ex] . 